\title{
Your error's got me feeling - how empathy relates to the electrophysiological correlates of performance monitoring
}

\section{Patrizia Thoma* and Christian Bellebaum}

Department of Neuropsychology, Institute of Cognitive Neuroscience, Ruhr-University Bochum, Bochum, Germany

Edited by:

Hauke R. Heekeren, Freie Universität

Berlin, Germany

\section{Reviewed by:}

Grit Hein, University of Zurich,

Switzerland

Sid Segalowitz, Brock University,

Canada

\section{*Correspondence:}

Patrizia Thoma, Department of Neuropsychology, Institute of

Cognitive Neuroscience,

Ruhr-University Bochum,

Universitätsstraße 150, 44780

Bochum, Germany.

e-mail: patrizia.thoma@rub.de

\begin{abstract}
The error-related and feedback-related negativities (ERN and FRN) represent negative event-related potentials associated with the processing of errors and (negative) response outcomes. The neuronal source of these components is considered to be in the anterior cingulate cortex (ACC). Monitoring one's own behavior and the impact it may have on other people or observing other individuals perform and receive feedback for their actions may also engage empathy-related processes. Empathy is conceived of as a multifaceted construct involving both cognitive and affective components, partly also supported by the ACC. The present mini-review aims to summarize the sparse database linking the electrophysiological correlates of performance monitoring to empathy. While most studies so far provide largely indirect evidence for such an association - e.g., by pointing toward altered ERN/FRN signaling in populations characterized by deviations in empathic responding - fewer investigations establish more explicit links between the two concepts. The relationship between state and, less consistently, trait measures of empathy and action monitoring might be more pronounced for observational than for active participation.
\end{abstract}

Keywords: error negativity, feedback negativity, empathy, perspective taking, observation learning

\section{INTRODUCTION}

The capacity to modify our behavior based on the feedback that we receive for our actions forms an integral part of our everyday life. It enables us to flexibly adapt to distinct environments characterized by different response-outcome contingencies. However, it is not only active learning that allows us to adjust our behavior but also the observation of other individuals being rewarded or punished for their actions. The evaluation of the affective consequences of the outcomes for the observed person and for oneself might involve empathy-related processes. The mirror neuron system, activated during self-performed but also observed actions, is thought to support both observational learning and our ability to resonate with other people's emotions (Gallese, 2003; Gallese et al., 2004). In our mini-review, we will consider empirical evidence for a link between action monitoring and empathy. Given space limitations, regarding the former, we will focus on the error-related negativity (ERN) and the feedback-related negativity (FRN) as electrophysiological correlates of action monitoring in the brain. The ERN and FRN components as well as current empathy concepts will be briefly introduced before we move on to studies linking these concepts.

\section{ACTION MONITORING: THE ERROR- AND FEEDBACK-RELATED NEGATIVITIES}

In event-related potential (ERP) studies, characteristic patterns of activity at fronto-central scalp electrodes have been associated with the monitoring of performance. While the response-locked ERN represents a negative deflection peaking within $100 \mathrm{~ms}$ after error commission (Falkenstein et al., 1991; Gehring et al., 1993), the FRN reaches a maximum after $200-300 \mathrm{~ms}$ following stimulus onset and is more pronounced for unfavorable as opposed to favorable performance feedback (Gehring and Willoughby, 2002; Nieuwenhuis et al., 2004). The neuronal generator for both components is assumed to be in the anterior cingulate cortex (ACC; Dehaene et al., 1994; Gehring and Willoughby, 2002), a region which has been related to various aspects of cognitive and emotional control [see the reviews by Allman et al., 2001; Van Veen and Carter, 2002; Rushworth et al., 2004; for evidence from functional magnetic resonance imaging (fMRI) studies]. The most common model explaining the functional significance of the ERN and FRN is the reinforcement learning theory (Holroyd and Coles, 2002). Within this framework, errors are conceptualized as "worse than expected outcomes" or negative "prediction errors," leading to an attenuation of phasic dopamine activity in the mesolimbic reward system. Unexpected reward (i.e., a positive prediction error), on the other hand, has been associated with increased phasic dopaminergic signaling. This signal is thought to guide action selection by the ACC, which will either be disinhibited or inhibited, affecting the probability with which an action that has or has not been reinforced in the past will be shown in the future. Alternative theories hold that the ERN reflects the motivational salience attributed to errors and the FRN the motivational and affective evaluation of outcomes (e.g., Gehring and Willoughby, 2002; Yeung et al., 2005), rendering it plausible that empathy might also play a role. This notion is further supported by a hypothesized link between dopaminergic prediction error signaling and context-dependent updating of our representations of other people's emotional states (Abu-Akel and Shamay-Tsoory, 2011).

MONITORING THE EMOTIONAL STATES OF OTHERS: EMPATHY

Empathy broadly refers to the capacity to respond to the emotional experiences of someone else. It is thought of as a multidimensional 
construct involving at least a cognitive component enabling an individual to understand another person's emotional perspective and an affective component based on the ability to affectively share and respond to the emotional experiences of others (Shamay-Tsoory, 2011). Empathic responding is modulated by the context of the interpersonal interaction and characteristics of the observer or the observed person (Hein and Singer, 2008), mediated by executive mechanisms which also keep track of the emotions' source (self vs. other), delineating empathy from pure emotional contagion (Decety and Lamm, 2006). The ventromedial and dorsomedial prefrontal cortices have been associated with cognitive empathy. Affective empathy might partly rely on more simple mechanisms such as emotion recognition, and on shared representations of affective experiences. The inferior frontal gyrus, inferior parietal lobe, anterior insula, and ACC have been linked to affective empathy, with the latter two structures playing a pivotal role in the "empathy for pain" network (see review by Shamay-Tsoory, 2011), although some argue that the anterior insula is more important than the ACC (Gu et al., 2010b). Electrophysiological evidence has repeatedly related state and trait empathic responding to enhanced mu/alpha suppression (e.g., Yang et al., 2009; Perry et al., 2010; Woodruff et al., 2011) and to a modulation of early fronto-central and late centro-parietal ERP amplitudes, partially affecting time windows, in which the ERN/FRN typically occur (Decety et al., 2010; Li and Han, 2010). Although most probably working in concert in most everyday situations, cognitive and affective empathy components can be impaired independently, e.g., in psychiatric disorders like autism (Dziobek et al., 2008), alcoholism (Maurage et al., 2011) and borderline personality disorder (Harari et al., 2010).

\section{INDIRECT EVIDENCE FOR A RELATIONSHIP BETWEEN EMPATHY AND THE ERN/FRN REPRESENTATION OF AVERSIVE EMOTIONAL STATES}

Given the prominent role of the ACC in cognitive and emotional control, it is not surprising that this structure has been related to empathy, particularly in response to aversive emotional states such as physical (Singer et al., 2004) and social pain (Eisenberger and Lieberman, 2004; Krach et al., 2011). Evidence from fMRI studies consistently suggests an overlap between the ACC activation during the first-hand experience of pain or other aversive emotions and during the mere observation of someone else experiencing these events with the strength of this overlapping ACC activity correlating positively with self-reported trait empathy (e.g., Singer et al., 2004; Krach et al., 2011). As mentioned previously, the FRN might also reflect the affective evaluation of negative performance feedback (Gehring and Willoughby, 2002; Yeung et al., 2005), potentially evoking aversive emotions. Transient negative affect and negative affect-related personality traits modulate the FRN and ERN. An enhanced FRN to negative but not to positive or neutral feedback has been related to increased state negative affect and anxiety (Gu et al., 2010a; Santesso et al., 2011) and clinical depression (Mies et al., 2011). Even with depression and anxiety being controlled for, the FRN remained increased in patients with remitted depression (Santesso et al., 2008). However, there are also reports of FRN reductions in association with depressive symptoms (Foti and Hajcak, 2009). Similarly, the ERN amplitude seems to be enhanced in participants with obsessive-compulsive disorder (Xiao et al., 2011), generalized anxiety disorder (Weinberg et al., 2010), and remitted clinical depression (Georgiadi et al., 2011), but reduced during severe depressive episodes (Ruchsow et al., 2004, 2006) with impaired differentiation between errors and correct responses (Olvet et al., 2010). The relationship between the ERN and negative affect seems to be further modulated by factors like psychomotor retardation (Schrijvers et al., 2008), perfectionism (Schrijvers et al., 2010), and neuroticism (Olvet and Hajcak, 2011). Healthy individuals learning better from negative than positive feedback also show increased ERN and FRN signaling (Frank et al., 2005). Overall, enhancement of these components in association with negative affect might point toward a hypervigilant ACC action monitoring system. Interestingly, individuals with clinical depression appear to show increased self-reported trait affective empathy (O'Connor et al., 2002; Thoma et al., 2011), indirectly highlighting an association between a hypervigilant action monitoring system, as indexed by the ERN/FRN, on the one hand and enhanced affective empathic responding on the other.

\section{ALTERED ACTION MONITORING IN POPULATIONS EXHIBITING ABNORMAL EMPATHIC RESPONDING}

The electrophysiological correlates of action monitoring are also altered in other populations typically exhibiting abnormal empathic responding. Diminished ERN amplitudes have been reported in individuals with autism spectrum disorder (Vlamings et al., 2008; Sokhadze et al., 2010; South et al., 2010), a population characterized by below average empathy (Baron-Cohen, 2010), possibly particularly regarding cognitive empathy and less so in terms of impaired affective empathy (Dziobek et al., 2008). Reduced ACC activity has been associated with attenuated ERN amplitudes, more severe social impairment and more pronounced psychopathology in adults and children with autism (Henderson et al., 2006; Santesso et al., 2010). On the other hand, the FRN was comparable in individuals with autism and controls, suggesting that the patients might primarily have difficulty with internal, more abstract regulation of performance and less so with feedback processing (Larson et al., 2011). Compared with autism, psychopathy has been associated with the reverse pattern of relatively intact or even superior cognitive and diminished affective empathy (Blair, 2008). While some authors have found reduced ERN, but intact FRN amplitudes (von Borries et al., 2010), others did not find any ERN changes (Brazil et al., 2009) in incarcerated, violent offenders with psychopathy. As these individuals are frequently involved in physical fights, potential previous head injury may confound interpretation of results. Munro et al. (2007a,b) controlled for this and reported reduced ERN and N2 amplitudes following errors in a flanker task with emotional faces but not with neutral letter stimuli, which illustrates an interaction of personality and context on error-related brain activation. Interestingly, compared with healthy controls, offenders with psychopathy performing a social flanker task showed similar ERN amplitudes during active performance, and diminished amplitudes when observing the performance of another individual (Brazil et al., 2011). This suggests a relatively specific impairment of other-related performance monitoring and possibly lower concern about other people's actions in this population. 


\section{ACTION MONITORING AND EMPATHY IN OBSERVATIONAL LEARNING}

The findings by Brazil et al. (2011) support the relationship between action monitoring and empathy playing a pivotal role in observation situations. The "observational ERN" or oERN reflects similar underlying neural mechanisms as the ERN elicited by active learning, although the peak of the former component seems to occur later and with an attenuated amplitude (van Schie et al., 2004). Similarly, the observational FRN (oFRN) is somewhat reduced in magnitude relative to the active FRN (Bellebaum et al., 2010). fMRI studies have confirmed that overlapping networks encompassing the dorsal ACC, the orbitofrontal cortex, the posterior medial frontal cortex, and supplementary motor regions mirror responses to one's own and to other people's errors (Shane et al., 2008; Brazil et al., 2011). This resembles evidence of overlapping ACC activations for one's own emotional experiences and during the observation of similar emotions in others, as cited above. Witnessing another individual's actions, the observer may rely on cognitive and affective empathy to infer how the other person might feel about her outcomes and what these might entail for one's own performance and outcomes. To date, few studies investigated these associations in observational learning, either indirectly or directly.

Based on the reasoning that empathic responding and the associated neural representations of other people's emotional states might be more pronounced toward individuals we feel emotionally closest to (e.g., Singer et al., 2004), a modulation of the ERN/FRN by the relationship between performer and observer might partly reflect empathy-related processes. While larger perceived similarity between observer and performer has been associated with a decreased oERN when observing confederates perform a flanker task (Carp et al., 2009), a more pronounced oFRN has been reported for participants observing friends vs. strangers complete a Stroop task, with the effect being mediated by the degree to which participants included the observed person in their self-concept (Kang et al., 2010). The fact that the participants' real-life friends were involved might have increased the probability of empathic reactions modulating the oFRN, while in the former study, larger perceived similarity with strangers might not have sufficed to do so. Decreased oERN amplitudes might even mirror the tendency to underestimate error commission by similar others.

According to Marco-Pallares et al. (2010), two different processes may affect the neural signal corresponding to the processing of observed response feedback: one might evaluate the consequences for oneself, while an empathy-related process might evaluate the outcome for the observed person. Depending on the social context, one or the other process might prevail and both may be modulated by different factors. In the betting task these authors used, a "neutral" observer group merely observed a performer's action; for a "parallel" group, losses or wins of the performer entailed similar outcomes for the observer, and in a "reverse" group, losses and wins of the performer signaled reverse outcomes for the observer. Participants showed a pronounced FRN to losses vs. gains, both as active players and as "neutral" or "parallel” observers. In the "reverse" group, however, an oFRN was elicited only in response to wins of the performer corresponding to losses for the observer. Similarly, active participation in a task may elicit competitive feelings, highlighting the need to evaluate outcome-related consequences for oneself and attenuating empathic responding toward the observed competitor. Accordingly, Ma et al. (2011) reported that an increased oFRN to a friend's relative to a stranger's performance could only be observed if the observer was not actively involved in the game.

\section{STUDIES ASSESSING BOTH EMPATHY AND THE ERN/FRN}

In contrast to the studies reported in the previous paragraphs, some authors used self-report measures of state or trait empathy allowing for a more direct investigation of the relationship between empathy and the ERN/FRN, although overall the result pattern does not appear consistent as yet.

Complementing the Ma et al. (2011) findings, Koban et al. (2010) showed that when participants' attentional resources were taken up by focusing on their own actions, the ERN during active learning was unaffected by a cooperative vs. competitive social context. In an observation condition, participants showed an "early" oERN after 125-145 ms during cooperation and a "late" oERN (280-320 ms) during competition. Trait empathy was unrelated to any of these components, but state measures of rivalry and competition toward the observed player were associated with a diminished early oERN, while the late oERN was smaller for participants who felt more sympathy and friendship toward the co-player. Having their participants play a competitive card game, Yamada et al. (2011) found larger FRN amplitudes on trials signaling "gain" for the participant and simultaneous "loss" for the confederate player (incongruent condition) relative to trials where both opponents lost (congruent condition), interpreting this as an effect of "counterempathy" or "schadenfreude." Larger FRN differences (incongruent-congruent loss) were related to higher subjective ratings of pleasantness about one's own winnings, but not to trait empathy. Only male participants were investigated, and there is evidence that gender may modulate empathy-related ACC activation (Singer et al., 2006) and the neural correlates of action monitoring in competitive situations. In a gambling task, where one player's monetary gain resulted in the opponent's loss, perception of the opponent's negative outcome elicited a small but discernible oFRN (loss-gain) in female, but not in male participants, even if the other individual's loss incurred wins for them (Fukushima and Hiraki, 2006). The authors attributed this to a more pronounced tendency of women to feel empathy for their opponents. Overall, the more the participants felt empathic concern about the opponent's outcomes, the less the oFRN diminished. Habitual tendencies to empathize and systemize (i.e., to focus on the analysis of physical objects and systems; Baron-Cohen et al., 2003) were also assessed. A higher "empathizing minus systemizing" score was negatively related to the amplitude of the oFRN, but not of the active FRN. The authors concluded that individual differences in empathy-related neural activity are best illustrated as a ratio between empathetic and non-empathetic (systemizing) functions.

Further support for the notion that the oFRN might be modulated by empathic responding specifically characterizing human interactions comes from a later study by Fukushima and Hiraki (2009). Participants performed actively, but also observed the performance of real-life friends or computer players, with the 
outcomes of the players being unrelated to each other. A significant oFRN was elicited only when humans were observed. Larger oFRN amplitudes were associated with higher dispositional cognitive and affective empathy, while there were no such relationships in the "computer player" condition. During active performance, higher cognitive empathy and smaller FRN amplitudes were marginally significantly correlated. This illustrates that while empathy might positively affect the monitoring of other people's actions, the tendency to habitually focus on other people's emotions might actually disrupt the monitoring of one's own performance. Depending on stimulus-feedback contingencies, this might also apply to observational learning. Kobza et al. (2011) had participants observe virtual others receiving positive or negative feedback for choosing between two symbols. The probability of positive feedback varied for different stimuli. Higher trait affective empathy was associated with poorer performance and higher trait cognitive empathy with smaller oFRN differences (positivenegative feedback) only when contingencies were most difficult to learn and feedback difficult to predict. The authors suggest that highly empathic individuals might tend to focus on the observed person's choice behavior rather than on response feedback contingencies to make sense of unpredictable feedback. Together with cognitive resources being taken up by attending to the emotional consequences for the observed person, this might particularly disrupt the learning of difficult associations. An alternative interpretation was suggested in an fMRI investigation by NewmanNorlund et al. (2009) who reported an association between higher trait empathic concern and weaker ventral ACC activation following error observation. According to the authors, empathic concern may also represent a disposition to regulate negative affect elicited by the observation of other people committing errors that might be relevant for oneself (in this case missed penalty shots of a soccer club one does or does not support). In cases where the observed errors might lack significance for the observer, empathic concern and ventral ACC activity seem to be associated positively (Shane et al., 2009).

While in the ERP studies presented earlier, ERN/FRN amplitudes correlated more consistently with state rather than with trait measures of empathy-related affective responding, FRN differences were associated with trait empathy in the two latter studies. Based on the evidence presented so far, it is difficult to decide which factors contributed to the inconsistencies. Note that at least in the Kobza et al. (2011) study, participants actually had to transfer the knowledge they acquired by observation to their own performance assessed in subsequent active test trials. This might have induced highly empathic individuals to try to benefit from the other person's coping with an ambiguous feedback situation to guide their own actions. Other studies focusing on active learning yielded significant correlations of the ERN with trait empathy. Santesso and Segalowitz (2009) reported significant associations between

\section{REFERENCES}

Abu-Akel, A., and Shamay-Tsoory, S. (2011). Neuroanatomical and neurochemical bases of theory of mind. Neuropsychologia 49, 2971-2984.

increased risk taking propensity and diminished ERN amplitudes and between higher trait empathy and larger ERN amplitudes in adolescents performing a flanker task. While high risk takers might not care about their errors and/or show diminished ability to learn from negative feedback, highly empathic individuals might be implicitly more concerned about the impact their actions might have on others. As empathy and risk taking were not correlated, they account for separate variance in the ERN. Larson et al. (2010) confirmed the association between larger ERN amplitudes and higher dispositional empathy, controlling for state negative affect. According to these authors, one construct that may explain the relationship between ERN and empathy might be vigilance to one's own performance and to the environment. Alternatively, both empathy and the ERN might be related to caring about positive or negative outcomes of one's own or other people's behavior.

\section{CONCLUSION}

Taken together, the evidence available so far points toward an association between empathy-related affective responding and the ERN/FRN components as electrophysiological correlates of action monitoring. The exact nature of the relationship is subject to modulation depending on state (negative) affect, personality and psychopathology, the type of learning (active or observational), gender, the specific stimulus-feedback contingencies and the interactive context (competitive vs. cooperative, relationship between performer and observer) among other factors. Currently, few studies explicitly assessed self-reported state or trait empathy in association with the ERN/FRN. Although part of the evidence nicely supports the association between ACC, empathy and ERN/FRN, some inconsistencies have to be borne in mind. For instance, while in fMRI studies, it is rather the rostral ACC that has been associated with error monitoring (Van Veen and Carter, 2002), mid-ACC (Lamm et al., 2011), or anterior insular activity (Gu et al., 2010b) seems to be more strongly related to empathy. On the other hand, trait empathy was found to relate to ventral ACC activity elicited following error observation, which possibly reflects affective aspects of error processing (Newman-Norlund et al., 2009). Future studies should try to more precisely disentangle the nature of the relationship between distinct empathy components and the ERN/FRN.

\section{ACKNOWLEDGMENTS}

Christian Bellebaum was supported by the Ministry of Innovation, Science and Research of the federal state of NordrheinWestfalen, Germany (Ministeriumfür Innovation, Wissenschaft und Forschung des Landes Nordrhein-Westfalen; MIWF - grant number 334-4). This work was also supported by the German Research Foundation (Deutsche Forschungsgemeinschaft - DFG), grant number TH 1535/2-1, awarded to Patrizia Thoma.

Baron-Cohen, S. (2010). Empathizing, systemizing, and the extreme male brain theory of autism. Prog. Brain Res. 186, 167-175.

Baron-Cohen, S., Richler, J., Bisarya, D., Gurunathan, N., and Wheelwright,
S. (2003). The systemizing quotient: an investigation of adults with Asperger syndrome or highfunctioning autism, and normal sex differences. Philos. Trans. R. Soc. Lond. B Biol. Sci. 358, 361-374. 
Bellebaum, C., Kobza, S., Thiele, S., and Daum, I. (2010). It was not MY fault: event-related brain potentials in active and observational learning from feedback. Cereb. Cortex 20, 2874-2883.

Blair, R. J. (2008). Fine cuts of empathy and the amygdala: dissociable deficits in psychopathy and autism. Q. J. Exp. Psychol. (Colchester) 61, 157-170.

Brazil, I. A., De Bruijn, E. R., Bulten, B. H., von Borries, A. K., van Lankveld, J. J., Buitelaar, J. K., and Verkes, R. J. (2009). Early and late components of error monitoring in violent offenders with psychopathy. Biol. Psychiatry 65, 137-143.

Brazil, I. A., Mars, R. B., Bulten, B. H., Buitelaar, J. K., Verkes, R. J., and De Bruijn, E. R. (2011). A neurophysiological dissociation between monitoring one's own and others' actions in psychopathy. Biol. Psychiatry 69, 693-699.

Carp, J., Halenar, M. J., Quandt, L. C., Sklar, A., and Compton, R. J. (2009). Perceived similarity and neural mirroring: evidence from vicarious error processing. Soc. Neurosci. 4, 85-96.

Decety, J., and Lamm, C. (2006). Human empathy through the lens of social neuroscience. ScientificWorldJournal 6, 1146-1163.

Decety, J., Yang, C. Y., and Cheng, Y. (2010). Physicians down-regulate their pain empathy response: an event-related brain potential study. Neuroimage 50, 1676-1682.

Dehaene, S., Posner, M. I., and Tucker, D. M. (1994). Localization of an neural system for error detection and compensation. Psychol. Sci. 5, 303-305.

Dziobek, I., Rogers, K., Fleck, S., Bahnemann, M., Heekeren, H. R., Wolf, O. T., and Convit, A. (2008). Dissociation of cognitive and emotional empathy in adults with Asperger syndrome using the Multifaceted Empathy Test (MET). J. Autism Dev. Disord. 38, 464-473.

Eisenberger, N. I., and Lieberman, M. D. (2004). Why rejection hurts: a common neural alarm system for physical and social pain. Trends Cogn. Sci. (Regul. Ed.) 8, 294-300.

Falkenstein, M., Hohnsbein, J., Hoormann, J., and Blanke, L. (1991). Effects of crossmodal divided attention on late ERP components. II. Error processing in choice reaction tasks. Electroencephalogr. Clin. Neurophysiol. 78, 447-455.

Foti, D., and Hajcak, G. (2009). Depression and reduced sensitivity to nonrewards versus rewards: evidence from event-related potentials. Biol. Psychol. 81, 1-8.

Frank, M. J., Woroch, B. S., and Curran, T. (2005). Error-related negativity predicts reinforcement learning and conflict biases. Neuron 47, 495-501.

Fukushima, H., and Hiraki, K. (2006). Perceiving an opponent's loss: gender-related differences in the medial-frontal negativity. Soc. Cogn. Affect. Neurosci. 1, 149-157.

Fukushima, H., and Hiraki, K. (2009). Whose loss is it? Human electrophysiological correlates of non-self reward processing. Soc. Neurosci. 4, 261-275.

Gallese, V. (2003). The manifold nature of interpersonal relations: the quest for a common mechanism. Philos. Trans. R. Soc. Lond. B Biol. Sci. 358, 517-528.

Gallese, V., Keysers, C., and Rizzolatti, G. (2004). A unifying view of the basis of social cognition. Trends Cogn. Sci. (Regul. Ed.) 8, 396-403.

Gehring, W. J., Goss, G. M. G. H. G., Meyer, D. E., and Donchin, E. (1993). A neural system for error detection and compensation. Psychol. Sci. 4 385-390.

Gehring, W. J., and Willoughby, A. R. (2002). The medial frontal cortex and the rapid processing of monetary gains and losses. Science 295, 2279-2282.

Georgiadi, E., Liotti, M., Nixon, N. L., and Liddle, P. F. (2011). Electrophysiological evidence for abnormal error monitoring in recurrent major depressive disorder. Psychophysiology 48, 1192-1202.

Gu, R., Ge, Y., Jiang, Y., and Luo, Y. J. (2010a). Anxiety and outcome evaluation: the good, the bad and the ambiguous. Biol. Psychol. 85, 200-206.

Gu, X., Liu, X., Guise, K. G., Naidich, T. P., Hof, P. R., and Fan, J. (2010b). Functional dissociation of the frontoinsular and anterior cingulate cortices in empathy for pain. J. Neurosci. 30, 3739-3744.

Harari, H., Shamay-Tsoory, S. G., Ravid, M., and Levkovitz, Y. (2010). Double dissociation between cognitive and affective empathy in borderline personality disorder. Psychiatry Res. $175,277-279$.

Hein, G., and Singer, T. (2008). I feel how you feel but not always: the empathic brain and its modulation. Curr. Opin. Neurobiol. 18, 153-158.

Henderson, H., Schwartz, C., Mundy, P., Burnette, C., Sutton, S., Zahka, N. and Pradella, A. (2006). Response monitoring, the error-related negativity, and differences in social behavior in autism. Brain Cogn. 61, 96-109.

Holroyd, C. B., and Coles, M. G. (2002). The neural basis of human error processing: reinforcement learning, dopamine, and the errorrelated negativity. Psychol. Rev. 109 679-709.

Kang, S. K., Hirsh, J. B., and Chasteen, A. L. (2010). Your mistakes are mine: self-other overlap predicts neural response to observed errors. J. Exp. Soc. Psychol. 46, 229-232.

Koban, L., Pourtois, G., Vocat, R., and Vuilleumier, P. (2010). When your errors make me lose or win: eventrelated potentials to observed errors of cooperators and competitors. Soc. Neurosci. 5, 360-374.

Kobza, S., Thoma, P., Daum, I., and Bellebaum, C. (2011). The feedbackrelated negativity is modulated by feedback probability in observational learning. Behav. Brain Res. 225, 396-404.

Krach, S., Cohrs, J. C., Echeverria Loebell, N. C., Kircher, T., Sommer, J., Jansen, A., and Paulus, F. M. (2011). Your flaws are my pain: linking empathy to vicarious embarrassment. PLoS ONE 6, e18675. doi:10.1371/journal.pone.0018675

Lamm, C., Decety, J., and Singer, T. (2011). Meta-analytic evidence for common and distinct neural networks associated with directly experienced pain and empathy for pain. Neuroimage 54, 2492-2502.

Larson, M. J., Fair, J. E., Good, D. A., and Baldwin, S. A. (2010). Empathy and error processing. Psychophysiology 47, 415-424.

Larson, M. J., South, M., Krauskopf, E., Clawson, A., and Crowley, M. J. (2011). Feedback and reward processing in high-functioning autism. Psychiatry Res. 187, 198-203.

Li, W., and Han, S. (2010). Perspective taking modulates event-related potentials to perceived pain. $\mathrm{Neu}$ rosci. Lett. 469, 328-332.

Ma, Q., Shen, Q., Xu, Q., Li, D., Shu, L., and Weber, B. (2011). Empathic responses to others' gains and losses: an electrophysiological investigation. Neuroimage 54, 2472-2480.

Marco-Pallares, J., Kramer, U. M., Strehl, S., Schroder, A., and Munte, T. F. (2010). When decisions of others matter to me: an electrophysiological analysis. BMC Neurosci. 11, 86 . doi:10.1186/1471-2202-11-86

Maurage, P., Grynberg, D., Noel, X., Joassin, F., Philippot, P., Hanak, C., Verbanck, P., Luminet, O., de Timary,
P., and Campanella, S. (2011). Dissociation between affective and cognitive empathy in alcoholism: a specific deficit for the emotional dimension. Alcohol. Clin. Exp. Res. 35, 1662-1668.

Mies, G. W., van der Veen, F. M., Tulen, J. H., Birkenhager, T. K., Hengeveld, M. W., and van der Molen, M. W. (2011). Drug-free patients with major depression show an increased electrophysiological response to valid and invalid feedback. Psychol. Med. 41, 2515-2525.

Munro, G. E., Dywan, J., Harris, G. T., McKee, S., Unsal, A., and Segalowitz, S. J. (2007a). ERN varies with degree of psychopathy in an emotion discrimination task. Biol. Psychol. 76, 31-42.

Munro, G. E., Dywan, J., Harris, G. T., McKee, S., Unsal, A., and Segalowitz, S. J. (2007b). Response inhibition in psychopathy: the frontal N2 and P3. Neurosci. Lett. 418, 149-153.

Newman-Norlund, R. D., Ganesh, S., van Schie, H. T., De Bruijn, E R., and Bekkering, H. (2009). Selfidentification and empathy modulate error-related brain activity during the observation of penalty shots between friend and foe. Soc. Cogn. Affect. Neurosci. 4, 10-22.

Nieuwenhuis, S., Holroyd, C. B., Mol, N., and Coles, M. G. (2004). Reinforcement-related brain potentials from medial frontal cortex: origins and functional significance. Neurosci. Biobehav. Rev. 28, 441-448.

O'Connor, L. E., Berry, J. W., Weiss, J., and Gilbert, P. (2002). Guilt, fear, submission, and empathy in depression. J. Affect. Disord. 71, 19-27.

Olvet, D. M., and Hajcak, G. (2011). The error-related negativity relates to sadness following mood induction among individuals with high neuroticism. Soc. Cogn. Affect. Neurosci. 7, 289-295.

Olvet, D. M., Klein, D. N., and Hajcak, G. (2010). Depression symptom severity and error-related brain activity. Psychiatry Res. 179, 30-37.

Perry, A., Bentin, S., Bartal, I. B., Lamm, C., and Decety, J. (2010). "Feeling" the pain of those who are different from us: modulation of EEG in the mu/alpha range. Cogn. Affect. Behav. Neurosci. 10, 493-504.

Ruchsow, M., Herrnberger, B. Beschoner, P., Gron, G., Spitzer, M., and Kiefer, M. (2006). Error processing in major depressive disorder: evidence from event-related potentials. J. Psychiatr. Res. 40, 37-46.

Ruchsow, M., Herrnberger, B., Wiesend, C., Gron, G., Spitzer, M., and Kiefer, 
M. (2004). The effect of erroneous responses on response monitoring in patients with major depressive disorder: a study with eventrelated potentials. Psychophysiology 41, 833-840.

Rushworth, M. F., Walton, M. E., Kennerley, S. W., and Bannerman, D. M. (2004). Action sets and decisions in the medial frontal cortex. Trends Cogn. Sci. (Regul. Ed.) 8, 410-417.

Santesso, D. L., Bogdan, R., Birk, J. L., Goetz, E. L., Holmes, A. J., and Pizzagalli, D. A. (2011). Neural responses to negative feedback are related to negative emotionality in healthy adults. Soc. Cogn. Affect. Neurosci. doi:10.1093/scan/nsr054. [E-pub ahead of print].

Santesso, D. L., Drmic, I. E., Jetha, M. K., Bryson, S. E., Goldberg, J. O., Hall, G. B., Mathewson, K. J., Segalowitz, S. J., and Schmidt, L. A. (2010). An event-related source localization study of response monitoring and social impairments in autism spectrum disorder. Psychophysiology 48, 241-251.

Santesso, D. L., and Segalowitz, S. J. (2009). The error-related negativity is related to risk taking and empathy in young men. Psychophysiology 46, 143-152.

Santesso, D. L., Steele, K. T., Bogdan, R., Holmes, A. J., Deveney, C. M., Meites, T. M., and Pizzagalli, D. A. (2008). Enhanced negative feedback responses in remitted depression. Neuroreport 19, 1045-1048.

Schrijvers, D., De Bruijn, E. R., Maas, Y., De Grave, C., Sabbe, B. G., and Hulstijn, W. (2008). Action monitoring in major depressive disorder with psychomotor retardation. Cortex 44 , 569-579.
Schrijvers, D. L., De Bruijn, E. R., Destoop, M., Hulstijn, W., and Sabbe, B. G. (2010). The impact of perfectionism and anxiety traits on action monitoring in major depressive disorder. J. Neural Transm. 117, 869-880.

Shamay-Tsoory, S. G. (2011). The neural bases for empathy. Neuroscientist $17,18-24$

Shane, M. S., Stevens, M., Harenski, C. L., and Kiehl, K. A. (2008). Neural correlates of the processing of another's mistakes: a possible underpinning for social and observational learning. Neuroimage 42, 450-459.

Shane, M. S., Stevens, M. C., Harenski, C. L., and Kiehl, K. A. (2009). Double dissociation between perspectivetaking and empathic-concern as predictors of hemodynamic response to another's mistakes. Soc. Cogn. Affect. Neurosci. 4, 111-118.

Singer, T., Seymour, B., O’Doherty, J., Kaube, H., Dolan, R. J., and Frith, C. D. (2004). Empathy for pain involves the affective but not sensory components of pain. Science 303, 1157-1162.

Singer, T., Seymour, B., O’Doherty, J. P., Stephan, K. E., Dolan, R. J., and Frith, C. D. (2006). Empathic neural responses are modulated by the perceived fairness of others. Nature 439, 466-469.

Sokhadze, E., Baruth, J., El Baz, A., Horrell, T., Sokhadze, G., Carroll, T., Tasman, A., Sears, L., and Casanova, M. F. (2010). Impaired error monitoring and correction function in autism. J. Neurother. 14, 79-95.

South, M., Larson, M. J., Krauskopf, E., and Clawson, A. (2010). Error processing in high-functioning autism spectrum disorders. Biol. Psychol. 85 , 242-251.

Thoma, P., Zalewski, I., von Reventlow, H. G., Norra, C., Juckel, G., and Daum, I. (2011). Cognitive and affective empathy in depression linked to executive control. Psychiatry Res. 189, 373-378.

Van Veen, V., and Carter, C. S. (2002). The anterior cingulate as a conflict monitor: fMRI and ERP studies. Physiol. Behav. 77, 477-482.

van Schie, H. T., Mars, R. B., Coles, M. G., and Bekkering, H. (2004). Modulation of activity in medial frontal and motor cortices during error observation. Nat. Neurosci. 7 , 549-554.

Vlamings, P. H., Jonkman, L. M., Hoeksma, M. R., van Engeland, H., and Kemner, C. (2008). Reduced error monitoring in children with autism spectrum disorder: an ERP study. Eur. J. Neurosci. 28, 399-406.

von Borries, A. K., Brazil, I. A. Bulten, B. H., Buitelaar, J. K., Verkes, R. J., and De Bruijn, E. R. (2010). Neural correlates of errorrelated learning deficits in individuals with psychopathy. Psychol. Med. 40, 1559-1568.

Weinberg, A., Olvet, D. M., and Hajcak, G. (2010). Increased error-related brain activity in generalized anxiety disorder. Biol. Psychol. 85, 472-480.

Woodruff, C. C., Martin, T., and Bilyk, N. (2011). Differences in self- and other-induced $\mathrm{Mu}$ suppression are correlated with empathic abilities. Brain Res. 1405, 69-76.

Xiao, Z., Wang, J., Zhang, M., Li, H., Tang, Y., Wang, Y., Fan, Q., and Fromson, J. A. (2011). Errorrelated negativity abnormalities in generalized anxiety disorder and obsessive-compulsive disorder. Prog. Neuropsychopharmacol. Biol. Psychiatry 35, 265-272.

Yamada, M., Lamm, C., and Decety, J. (2011). Pleasing frowns, disappointing smiles: an ERP investigation of counterempathy. Emotion 11, 1336-1345.

Yang, C. Y., Decety, J., Lee, S., Chen, C., and Cheng, Y. (2009). Gender differences in the mu rhythm during empathy for pain: an electroencephalographic study. Brain Res. 1251, 176-184.

Yeung, N., Holroyd, C. B., and Cohen, J. D. (2005). ERP correlates of feedback and reward processing in the presence and absence of response choice. Cereb. Cortex 15 535-544.

Conflict of Interest Statement: The authors declare that the research was conducted in the absence of any commercial or financial relationships that could be construed as a potential conflict of interest.

Received: 13 February 2012; accepted: 26 April 2012; published online: 17 May 2012.

Citation: Thoma $P$ and Bellebaum $C$ (2012) Your error's got me feeling - how empathy relates to the electrophysiological correlates of performance monitoring. Front. Hum. Neurosci. 6:135. doi: 10.3389/fnhum.2012.00135

Copyright (C) 2012 Thoma and Bellebaum. This is an open-access article distributed under the terms of the Creative Commons Attribution Non Commercial License, which permits noncommercial use, distribution, and reproduction in other forums, provided the original authors and source are credited. 